\title{
Gibbs random graphs on point processes
}

\author{
Pablo A. Ferrari, ${ }^{1,4, a)}$ Eugene A. Pechersky, ${ }^{2, b)}$ Valentin V. Sisko, ${ }^{3, c)}$ and \\ Anatoly A. Yambartsev4,d) \\ ${ }^{1}$ Departamento de Matemática, Facultad de Ciencias Exactas y Naturales, Universidad de \\ Buenos Aires, Pabellón 1, Ciudad Universitaria, 1428 Buenos Aires, Argentina \\ ${ }^{2}$ Dobrushin Laboratory of Institute for Information Transmission Problems of Russian \\ Academy of Sciences, 19, Bolshoj Karetny, Moscow, Russia \\ ${ }^{3}$ Federal University of Fluminense, Institute of Matematics and Statistics, Rua Mário Santos \\ Braga, s/n, 24020-140 Niterói, RJ, Brazil \\ ${ }^{4}$ Department of Statistics, Institute of Mathematics and Statistics, University of São Paulo, \\ Rua do Matão 1010, CEP 05508-090, São Paulo SP, Brazil
}

(Received 3 March 2010; accepted 12 October 2010; published online 29 November 2010)

\begin{abstract}
Consider a discrete locally finite subset $\Gamma$ of $\mathbb{R}^{d}$ and the complete graph $(\Gamma, E)$, with vertices $\Gamma$ and edges $E$. We consider Gibbs measures on the set of sub-graphs with vertices $\Gamma$ and edges $E^{\prime} \subset E$. The Gibbs interaction acts between open edges having a vertex in common. We study percolation properties of the Gibbs distribution of the graph ensemble. The main results concern percolation properties of the open edges in two cases: (a) when $\Gamma$ is sampled from a homogeneous Poisson process; and (b) for a fixed $\Gamma$ with sufficiently sparse points. (c) 2010 American Institute of Physics. [doi:10.1063/1.3514605]
\end{abstract}

\section{INTRODUCTION}

Let a sample $\Gamma \subset \mathbb{R}^{d}$ of a point process be a locally finite set of $\mathbb{R}^{d}$. We consider an ensemble of graphs with vertices $\Gamma$ and random edges belonging to the set of unordered pairs of points in $\Gamma$. The edges can be open or closed and we study probability distributions on the set of configurations of open edges. The classical example is the Erdös-Rényi's random graph where each edge is open independently of the others with some probability, see Refs. 1 and 2. We introduce interactions between edges and/or vertices and study the associated Gibbs measures.

Given a configuration of open edges, two edges collide if both of them are open and they have a vertex in common. Monomers are those vertices that belong to no open edge. Each collision and each monomer contribute a positive energy; any open edge contributes a positive energy proportional to its length. The energy function $H$ is described explicitly in (1) later. The Gibbs measure associated with $H$ at inverse temperature $\beta>0$ gives more weight to configurations with few monomers, few (or no) collisions and short edges.

In Theorem 1, we give sufficient conditions on $\Gamma$ for the existence of an infinite volume Gibbs measure. An open edge not colliding with any other edge is called dimer. In Theorem 2, we show that the ground states for any locally finite configuration $\Gamma$ are composed only of monomers and dimers. Theorem 3 gives conditions for the uniqueness of the ground state. Theorem 4 shows that if $\Gamma$ is a sample of a homogeneous Poisson process with small density and low temperature, then there is no percolation for almost all $\Gamma$. Non-percolation in this context is the absence of an infinite sequence of colliding open edges a.s. with respect to the Gibbs measure. Theorem 5 proves that if $\Gamma$ is "sparse" and the temperature is sufficiently low, then there is no percolation a.s. with respect to

\footnotetext{
a)Electronic mail: pferrari@dm.uba.ar and pablo@ime.usp.br.

b)Electronic mail: pech@iitp.ru.

c) Electronic mail: valentin@mat.uff.br.

d)Electronic mail: yambar@ime.usp.br.
} 
the Gibbs measure. In particular for $\Gamma$ satisfying an $\varepsilon$-hard-core condition (that is, the ball of radius $\varepsilon$ around each point of $\Gamma$ has no other point of $\Gamma$ ) for some $\varepsilon>0$, there is no percolation.

\section{DEFINITIONS}

Let $\Gamma$ be a locally finite set of $\mathbb{R}^{d}$ and consider the complete graph $(\Gamma, E)$ with vertex set $\Gamma$ and edge set $E:=\left\{\gamma \gamma^{\prime}: \gamma, \gamma^{\prime} \in \Gamma\right\}$. The length of the edge $e=\gamma \gamma^{\prime}$, is denoted by $L(e):=\left|\gamma-\gamma^{\prime}\right|$. For a point $\gamma \in \Gamma$, let $S_{\gamma}:=\left\{\gamma \gamma^{\prime} \in E: \gamma^{\prime} \in \Gamma\right\}$, the set of edges incident on $\gamma$.

Let $\bar{\Omega}$ be the set of subsets of $E$. A configuration $\omega \in \bar{\Omega}$ defines the sub-graph $(\Gamma, \omega)$. Edges $e \in \omega$ are called open with respect to $\omega$. Edges $e \in E \backslash \omega$ are called closed with respect to $\omega$. When it is clear from the context we use the terms open and closed with no mention to the configuration $\omega$. The degree $d_{\gamma}(\omega)$ of a vertex $\gamma \in \Gamma$ is the number of open edges in $\omega$ containing $\gamma$ :

$$
d_{\gamma}(\omega):=\left|S_{\gamma} \cap \omega\right| \text {. }
$$

\section{A. Gibbs measures}

The set of $\omega \in \bar{\Omega}$ with finite-degree vertices is called

$$
\Omega:=\left\{\omega: \omega \in \bar{\Omega}, d_{\gamma}(\omega)<\infty \text { for all } \gamma\right\} .
$$

Our goal is to define a Gibbs distribution on $\Omega$ associated to the following formal Hamiltonian

$$
H(\omega):=\sum_{e \in \omega} L(e)+\sum_{\gamma \in \Gamma} \phi_{\gamma}(\omega),
$$

where $\phi_{\gamma}(\omega)$ is the contribution of monomers and interacting edges in $S_{\gamma}$ defined by

$$
\phi_{\gamma}(\omega):= \begin{cases}h_{0}, & \text { if } d_{\gamma}(\omega)=0 \\
0, & \text { if } d_{\gamma}(\omega)=1 \\
h_{1}\left(\begin{array}{c}
d_{\gamma}(\omega) \\
2
\end{array}\right), & \text { if } d_{\gamma}(\omega) \geq 2\end{cases}
$$

where $0<h_{0}<h_{1}$ are fixed parameters. The potential $\phi_{\gamma}(\omega)$ depends only on the degree $d_{\gamma}(\omega)$. Degree zero contributes $h_{0}$, degree 1 does not contribute and each pair of open edges incident on $\gamma$ contributes $h_{1}$.

The potential function $\phi_{\gamma}$ depends on infinitely many edges because to establish if $d_{\gamma}(\omega)=0$ we have to check that $e \in \omega$ for infinitely many edges $e \in S_{\gamma}$. However, the usual Gibbs construction (including existence theorem) for this case works without special considerations.

We define a family of finite volume Gibbs measures. For bounded $\Lambda \subset \mathbb{R}^{d}$ the set $\Gamma_{\Lambda}:=\Gamma \cap \Lambda$ is finite and so is $E_{\Lambda}:=\left\{\gamma \gamma^{\prime}: \gamma, \gamma^{\prime} \in \Gamma_{\Lambda}\right\}$. The Gibbs state $P_{\Lambda}$ on $\Omega_{\Lambda}:=\left\{\omega \in \bar{\Omega}: \omega \subset E_{\Lambda}\right\}$ with the zero boundary configuration is defined by

$$
\mathrm{P}_{\Lambda}(\omega):=\frac{\exp \left\{-\beta H_{\Lambda}(\omega)\right\}}{Z_{\Lambda}}, \quad \omega \in \Omega_{\Lambda},
$$

where the parameter $\beta$ is called inverse temperature, $Z_{\Lambda}$ is the normalizing constant and

$$
H_{\Lambda}(\omega):=\sum_{e \in \omega} L(e)+\sum_{\gamma \in \Gamma_{\Lambda}} \phi_{\gamma}(\omega), \quad \omega \in \Omega_{\Lambda} .
$$

Denote $E_{\Lambda}$ the expectation with respect to $P_{\Lambda}$.

We only consider Gibbs distributions $\mathrm{P}$ on $\bar{\Omega}$ associated with the formal Hamiltonian $H$ defined in (1) that can be constructed as a limit along subsequences of $\mathrm{P}_{\Lambda}, \Lambda \uparrow \mathbb{R}^{d}$, where $\mathrm{P}_{\Lambda}$ is defined in (3). Since $\bar{\Omega}$ is compact, $P$ exists but may have infinite-degree vertices. In Theorem 1 we show that under mild conditions on $\Gamma$, all vertex has finite degree with $P$-probability 1 . This implies that $P$ is concentrated on $\Omega$. Uniqueness of $P$ is not discussed in this article. 
The measure $\mathrm{P}_{\Lambda}$ can be seen as a measure on $\bar{\Omega}$ concentrating mass in the set $\Omega_{\Lambda}$.

\section{B. Percolation}

A path of length $n$ connecting $\gamma_{0}$ with $\gamma_{n}$ in the graph $(\Gamma, E)$ is a sequence of distinct vertices $\gamma_{0}, \ldots, \gamma_{n}$ and the edges connecting successive points. A path is open if all its edges $\gamma_{i} \gamma_{i+1}$ are open. A connected component of $\omega$ is a set of points that can be mutually connected by open paths and the open edges incident to those points. Maximal connected components of $\omega$ are called open clusters. The open cluster at $\gamma$, called $C_{\gamma}(\omega)$, is the maximal connected component containing $\gamma$. The vertex set of $C_{\gamma}(\omega)$ is the set of all vertices in $\Gamma$ which are connected to $\gamma$ by open paths and the edge set of $C_{\gamma}(\omega)$ is the set of edges of $\omega$ which join pairs of such vertices.

We give sufficient conditions for the absence of infinite clusters with $\mathrm{P}$ probability one; this is called no percolation.

\section{MAIN RESULTS}

\section{A. Existence}

Let $\alpha>0$. A point set $\Gamma$ is $\alpha$-homogeneous if for any $\gamma \in \Gamma$,

$$
T_{\gamma}(\alpha):=\sum_{\gamma^{\prime} \in \Gamma} e^{-\alpha L\left(\gamma \gamma^{\prime}\right)}<\infty .
$$

A point set $\Gamma$ is uniformly $\alpha$-homogeneous if

$$
T(\alpha):=\sup _{\gamma \in \Gamma} T_{\gamma}(\alpha)<\infty .
$$

If $\Gamma$ consists of hard core ball centers of a fixed radius then $\Gamma$ is uniformly $\alpha$-homogeneous for all $\alpha$; this is because the number of points grows polynomially with the distance while the weight decreases exponentially. Almost all sample $\Gamma$ from a Poisson process is $\alpha$-homogeneous for all $\alpha$ but uniformly $\alpha$-homogeneous for no $\alpha$; see Lemma 2.

Theorem 1: Take $0<h_{0}<h_{1}, \beta>0$ and a $\beta$-homogeneous $\Gamma$. Then, any Gibbs measure $\mathrm{P}$ at inverse temperature $\beta$ is concentrated on $\Omega$.

\section{B. Ground states}

A configuration $\widehat{\omega}$ is a local perturbation of $\omega \in \Omega$ if the symmetric difference $\widehat{\omega} \Delta \omega$ is a finite set. A configuration $\omega \in \Omega$ is a ground state if

$$
H(\widehat{\omega})-H(\omega) \geq 0
$$

for any local perturbation $\widehat{\omega}$ of $\omega$. The difference is well defined because all but a finite number of terms vanish.

The next result says that all ground states are composed by dimers and monomers.

Theorem 2: For any locally finite configuration $\Gamma$ and any $0<h_{0}<h_{1}$ there exists at least one ground state. If $\omega$ is a ground state then

$$
d_{\gamma}(\omega) \leq 1
$$

for every $\gamma \in \Gamma$. The length of any open edge in $\omega$ is less than $2 h_{0}$.

Let $\pi_{\lambda}$ be the distribution of a homogeneous Poisson process on $\mathbb{R}^{d}$ with rate $\lambda>0$.

Theorem 3: There exists $\lambda_{g}$ such that if $\lambda<\lambda_{g}$, then for $\pi_{\lambda}$-almost all $\Gamma$ there is only one ground state. 


\section{No percolation at low rate and temperature}

We consider the random graph as a dependent percolation model. ${ }^{4}$ For $\gamma \in \Gamma$, let $\left|C_{\gamma}(\omega)\right|$ be the number of vertices in $C_{\gamma}(\omega)$ and let $\theta_{\gamma}(\mathrm{P})$ be the probability that the open cluster at $\gamma$ is infinite:

$$
\theta_{\gamma}(\mathrm{P}):=\mathrm{P}\left(\left|C_{\gamma}(\omega)\right|=\infty\right) .
$$

We say that there is no percolation for $\mathrm{P}$ if $\theta_{\gamma}(\mathrm{P})=0$.

Take a Poisson process of rate $\lambda$ and a Gibbs state at inverse temperature $\beta$. We establish a $(\lambda, \beta)$-region where there is no percolation for $\mathrm{P}$ :

Theorem 4: If

$$
\lambda\left(2 h_{0}+\frac{\log 2}{\beta}\right) \leq 1
$$

then there is no percolation for $\mathrm{P}$ for $\pi_{\lambda}$-almost all $\Gamma$.

The next theorem is stronger but for more restricted sets $\Gamma$.

Theorem 5: If $\Gamma$ is such that

$$
\varepsilon:=T(\beta) \exp \left(-\beta\left(h_{1}-h_{0}\right)\right)<1,
$$

then there is no percolation for $\mathrm{P}$.

Remark 1: Fix $\Gamma, h_{0}$ and $h_{1}$. Then $T(\beta)$ is a non-increasing function of $\beta$. Therefore the inequality (8) is valid for all $\beta$ big enough.

\section{PROOFS}

Along this section $\Gamma$ is assumed $\beta$-homogeneous. Let $\mathrm{P}^{\circ}$ be the product measure on $\bar{\Omega}$ with marginals

$$
\mathrm{P}^{\circ}(e \in \omega)=\frac{e^{-\beta L(e)}}{e^{-\beta L(e)}+e^{-2 \beta h_{0}}} .
$$

Lemma 1: Any infinite volume Gibbs measure $\mathrm{P}$ is stochastically dominated by the product measure $\mathrm{P}^{\circ}$ : for any finite set of edges $\zeta \subset E$,

$$
\mathrm{P}(\omega \supset \zeta) \leq \mathrm{P}^{\circ}(\omega \supset \zeta)
$$

Proof: We start proving that for any bounded $\Lambda \subset \mathbb{R}^{d}, \mathrm{P}_{\Lambda}$ is stochastically dominated by $\mathrm{P}_{\Lambda}^{o}$ the product measure defined on $\Omega_{\Lambda}$. Holley Theorem says that if for all $\tilde{\omega} \subset E_{\Lambda}$

$$
\mathrm{p}:=\mathrm{P}_{\Lambda}(e \in \omega \mid \omega \backslash\{e\}=\tilde{\omega} \backslash\{e\}) \leq \mathrm{P}_{\Lambda}^{\mathrm{o}}(e \in \omega) .
$$

then $\mathrm{P}_{\Lambda}$ is stochastically dominated by $\mathrm{P}_{\Lambda}^{\mathrm{o}}$; see Theorem 4.8 in Ref. 3 . This is enough to prove the lemma because for finite $\zeta,\{\omega \supset \zeta\}$ is a cylinder set and the limit

$$
\begin{aligned}
\mathrm{P}(\omega \supset \zeta) & =\lim _{\Lambda \uparrow \mathbb{R}^{d}} \mathrm{P}_{\Lambda}(\omega \supset \zeta) \\
& \leq \lim _{\Lambda \uparrow \mathbb{R}^{d}} \mathrm{P}_{\Lambda}^{\mathrm{o}}(\omega \supset \zeta)=\mathrm{P}^{\mathrm{o}}(\omega \supset \zeta)
\end{aligned}
$$

holds along subsequences.

Now we prove (10). Let $e=\gamma_{1} \gamma_{2} \in E_{\Lambda}$. The conditional probability in (10) depends only on configurations on $\left(S_{\gamma_{1}} \cup S_{\gamma_{2}}\right) \cap E_{\Lambda} \backslash\{e\}$ :

$$
\mathrm{p}=\mathrm{P}_{\Lambda}\left(e \in \omega \mid \omega_{\gamma_{1}}=\tilde{\omega}_{\gamma_{1}}, \omega_{\gamma_{2}}=\tilde{\omega}_{\gamma_{2}}\right),
$$

where $\omega_{\gamma}=\omega \cap S_{\gamma} \cap E_{\Lambda} \backslash\{e\}$. Consider three cases: 
1. $\tilde{\omega}_{\gamma_{1}}=\tilde{\omega}_{\gamma_{2}}=\emptyset$,

3. $\tilde{\omega}_{\gamma_{1}} \neq \emptyset, \quad \tilde{\omega}_{\gamma_{2}}=\emptyset$ (and its symmetric version $\left.\tilde{\omega}_{\gamma_{1}}=\emptyset, \quad \tilde{\omega}_{\gamma_{2}} \neq \emptyset\right)$.

3. $\tilde{\omega}_{\gamma_{1}} \neq \emptyset, \tilde{\omega}_{\gamma_{2}} \neq \emptyset$.

Case 1: The edge $e$ is isolated of the rest, so the probability to be in $\omega$ is

$$
\mathrm{p}=\frac{e^{-\beta L(e)}}{e^{-\beta L(e)}+e^{-2 \beta h_{0}}}=\mathrm{P}^{\mathrm{o}}(e \in \omega) .
$$

Case 2: Let $m$ be the number of open edges in $\tilde{\omega}_{\gamma_{1}}$. If $e$ is open then it interacts with $m$ open edges:

$$
\begin{aligned}
\mathrm{p} & =\frac{e^{-\beta L(e)-\beta m h_{1}}}{e^{-\beta L(e)-\beta m h_{1}}+e^{-\beta h_{0}}} \\
& <\frac{e^{-\beta L(e)}}{e^{-\beta L(e)}+e^{-2 \beta h_{0}}}=\mathrm{P}^{\mathrm{o}}(e \in \omega) .
\end{aligned}
$$

Case 3: Let $m$ be the number of open edges in $\tilde{\omega}_{\gamma_{1}} \cup \tilde{\omega}_{\gamma_{2}}$. Then,

$$
\begin{aligned}
\mathrm{p} & =\frac{e^{-\beta L(e)-\beta m h_{1}}}{e^{-\beta L(e)-\beta m h_{1}}+1}<\frac{e^{-\beta L(e)}}{e^{-\beta L(e)}+1} \\
& <\frac{e^{-\beta L(e)}}{e^{-\beta L(e)}+e^{-2 \beta h_{0}}}=\mathrm{P}^{\mathrm{o}}(e \in \omega) .
\end{aligned}
$$

Proof of Theorem 1: $\quad$ Use Lemma 1 to dominate the degree of $\gamma$ as follows

$$
\begin{aligned}
\mathrm{E} d_{\gamma} & =\sum_{\gamma^{\prime} \in \Gamma} \mathrm{P}\left(\gamma \gamma^{\prime} \in \omega\right) \leq \sum_{\gamma^{\prime} \in \Gamma} \mathrm{P}^{\mathrm{o}}\left(\gamma \gamma^{\prime} \in \omega\right) \\
& =\sum_{\gamma^{\prime} \in \Gamma} \frac{e^{-\beta L\left(\gamma \gamma^{\prime}\right)}}{e^{-\beta L\left(\gamma \gamma^{\prime}\right)}+e^{-2 \beta h_{0}}} \leq \frac{T_{\gamma}(\beta)}{e^{-2 \beta h_{0}}}<\infty,
\end{aligned}
$$

because $\Gamma$ is $\beta$-homogeneous by hypothesis.

Lemma 2: Almost all samples $\Gamma$ from the Poisson process distribution $\pi_{\lambda}$ are $\alpha$-homogeneous for all $\alpha$.

Proof: Without losing generality assume $0 \in \Gamma$. Consider a sequence of hypercubes $\Lambda_{n}=$ $\left[-n^{1 / d}, n^{1 / d}\right]^{d}, n \geq 0$. Any ring $W_{n}=\Lambda_{n+1} \backslash \Lambda_{n}, n \geq 0$, has volume 2 . If $\gamma \in W_{n}$ then $L(0 \gamma) \geq$ $n^{1 / d}$ and

$$
\sum_{\gamma \in \Gamma} e^{-\alpha L(0 \gamma)} \leq \sum_{n=0}^{\infty}\left|\Gamma \cap W_{n}\right| \exp \left(-\alpha n^{(1 / d)}\right) .
$$

Since $\left|\Gamma \cap W_{n}\right|$ is a Poisson random variable with mean 2 for all $n$, the sum is finite $\pi_{\lambda}$-a.s. for any $\alpha>0$.

Proof of Theorem 2: Assume $\omega$ is a ground state and proceed by contradiction: assume that there exists a vertex $\gamma \in \Gamma$ such that $d_{\gamma}(\omega) \geq 2$. Let $\gamma \gamma^{\prime} \in \omega$. Let $\tilde{\omega}$ be the same as $\omega$ but without the edge $\gamma \gamma^{\prime}$ :

$$
\tilde{\omega}:=\omega \backslash\left\{\gamma \gamma^{\prime}\right\}
$$

Then,

$$
H(\omega)-H(\tilde{\omega})= \begin{cases}L\left(\gamma \gamma^{\prime}\right)+\left(d_{\gamma}(\tilde{\omega})+d_{\gamma^{\prime}}(\tilde{\omega})\right) h_{1} & \text { if } d_{\gamma^{\prime}}(\tilde{\omega}) \geq 1, \\ L\left(\gamma \gamma^{\prime}\right)+d_{\gamma}(\tilde{\omega}) h_{1}-h_{0} & \text { if } d_{\gamma^{\prime}}(\tilde{\omega})=0 .\end{cases}
$$

Since $0<h_{0}<h_{1}$, we have $H(\omega)-H(\tilde{\omega})>0$, which contradicts that $\omega$ is a ground state.

There are no edges in a ground state with length $L$ greater than $2 h_{0}$, since the energy of two monomers is $2 h_{0}<L$. 
Now we prove existence of at least one ground state. Let $\left(\Lambda_{n}\right)$ be a sequence of increasing cubes covering $\mathbb{R}^{d}=\bigcup_{n} \Lambda_{n}$. Let $\omega_{n}$ be a configuration in $\Lambda_{n}$ having the minimal energy over all configurations in $\Lambda_{n}$. There exists a subsequence $\left(\omega_{i}^{\prime}\right)$ of the sequence $\left(\omega_{n}\right)$ (that is $\left.\omega_{i}^{\prime}=\omega_{n_{i}}\right)$ such that there exists a limit $\lim _{i \rightarrow \infty} \omega_{i}^{\prime}(e)$ for every $e \in E$ (here $w(e)=1$ if $e \in \omega$ and $w(e)=0$ otherwise). Moreover, the sequence $\left(\omega_{i}^{\prime}\right)$ can be chosen in such a way that $\omega_{j}^{\prime}(e) \equiv$ const for all $j \geq i$ when $e \in E_{\Lambda_{i}}$. The configuration $\omega^{\prime}=\bigcup_{i} \omega_{i}^{\prime}$ is one of the ground states. To see it, let $\widehat{\omega}$ be a local perturbation of $\omega^{\prime}$. There exists $k$ such that $\widehat{\omega} \Delta \omega^{\prime} \subseteq E_{\Lambda_{k}}$. For $i>k$ let $\widehat{\omega}_{i}$ be the restriction of $\widehat{\omega}$ to $E_{\Lambda_{i}}$. Since $\widehat{\omega}_{i}$ is a perturbation of $\omega_{i}^{\prime}$,

$$
H_{\Lambda_{i}}\left(\widehat{\omega}_{i}\right)-H_{\Lambda_{i}}\left(\omega_{i}^{\prime}\right) \geq 0 .
$$

Since no $\omega_{i}$ has edges with a length greater than $2 h_{0}$, there exists $i_{1} \geq i_{0}$ such that

$$
H(\widehat{\omega})-H\left(\omega^{\prime}\right)=H_{\Lambda_{i_{1}}}\left(\widehat{\omega}_{i_{1}}\right)-H_{\Lambda_{i_{1}}}\left(\omega_{i_{1}}^{\prime}\right) \geq 0 .
$$

This proves that $\omega^{\prime}$ is a ground state.

Proof of Theorem 3: Let $\Gamma$ be a sample of $\pi_{\lambda}$, the law of a Poisson process with intensity $\lambda$. Let $U$ be the union of all circles of radius $h_{0}$ centered at the points of $\Gamma$. There exists a critical intensity $\lambda_{c}$ such that for any $\lambda<\lambda_{c}$ any maximal connected component of $U$ is bounded $\pi_{\lambda}$-a.s.; see Theorem 3.3 in Ref. 5. Thus, for $\lambda<\lambda_{c} \pi_{\lambda}$-almost-all $\Gamma$ is a union of finite clusters $\Gamma=\cup_{i=1}^{\infty} \Gamma_{i},\left|\Gamma_{i}\right|<\infty$, and for any $i \neq j$ and for any $\gamma \in \Gamma_{i}, \gamma^{\prime} \in \Gamma_{j}$ the distance $\left|\gamma-\gamma^{\prime}\right|>2 h_{0}$. There are open edges only inside the clusters $\Gamma_{i}$. Since $\Gamma_{i}$ are finite there exists a unique configuration in $\Gamma_{i}$ minimizing the energy.

The random connection model. Choose a point configuration $\Gamma$ with $\pi_{\lambda}$ and then the edges with $\mathrm{P}^{\mathrm{o}}$. The resulting random graph is called random-connection model with rate $\lambda$ and connection function

$$
g(x):=\frac{e^{-\beta x}}{e^{-\beta x}+e^{-2 \beta h_{0}}} .
$$

See Chapter 6 in Ref. 5. The connection function $g(x)$ is the probability that two points at distance $x$ be connected. To show Theorem 4 we will dominate our graph with this model. The next lemma gives a sufficient condition for non-percolation in the random connection model.

Lemma 3: $\quad$ In the region (7) for $\pi_{\lambda}$-almost all $\Gamma$ there is no percolation in the random-connection model with connection function (12).

Proof: $\quad$ Theorem 6.1 of Ref. 5 establishes that the random-connection model with connection function (12) does not percolate if

$$
\lambda \int_{0}^{\infty} g(x) \mathrm{d} x<1 .
$$

For any $\beta>0$ and any $h_{0}>0$ the integral of $g(x)$ in (13) is finite and equals

$$
\int_{0}^{2 h_{0}} \frac{e^{-\beta x}}{e^{-\beta x}+e^{-2 \beta h_{0}}} \mathrm{~d} x+\int_{2 h_{0}}^{\infty} \frac{e^{-\beta x}}{e^{-\beta x}+e^{-2 \beta h_{0}}} \mathrm{~d} x:=J_{1}(\beta)+J_{2}(\beta) .
$$

The first integral on the right side of the above equality is increasing and tends to $2 h_{0}$ as $\beta \rightarrow \infty$. The second integral tends to 0 as $\beta \rightarrow \infty$. Fix $\beta>0$ and choose $\lambda$ such that

$$
\lambda<\frac{1}{2 h_{0}+J_{2}(\beta)} \leq \frac{1}{\int g(x) \mathrm{d} x} .
$$

We obtain the lemma by computing $J_{2}(\beta)=\frac{\log 2}{\beta}$.

Proof of Theorem 4: Lemma 1 says that the Gibbs measure $\mathrm{P}$ is dominated by the product measure $P^{o}$ defined by (9). Lemma 3 implies that the product measure $P^{\circ}$ does not percolate under the conditions of the theorem. Thus the Gibbs measure P does not percolate for $\mu_{\lambda}-\mathrm{a} . \mathrm{s} . \Gamma$. 
Lemma 4: $\quad$ Let $B_{\gamma}$ and $D_{\gamma}$ be a partition of $S_{\gamma}$. Assume $B_{\gamma}$ is finite and that $\xi$ is a nonempty set of edges contained in $B_{\gamma}$. Then, for any $e \in D_{\gamma}$,

$$
\mathrm{P}\left(e \in \omega \mid \omega \cap B_{\gamma}=\xi\right) \leq \exp \left(-\beta L(e)-\beta\left(h_{1}-h_{0}\right)\right) .
$$

Proof: $\quad$ Consider any bounded $\Lambda \subset \mathbb{R}^{d}$ such that $\Lambda \supset B_{\gamma} \cup\{e\}$. Let

$$
\begin{aligned}
& A:=\left\{\omega \subset E_{\Lambda}: \omega \cap B_{\gamma}=\xi, e \in \omega\right\}, \\
& Z:=\sum_{\omega \subset E_{\Lambda}: \omega \cap B_{\gamma}=\xi} \exp \left(-\beta H_{\Lambda}(\omega)\right)
\end{aligned}
$$

and compute

$$
\begin{aligned}
& \mathrm{P}_{\Lambda}\left(e \in \omega \mid \omega \cap B_{\gamma}=\xi\right) \\
& \quad=\frac{1}{Z} \sum_{\omega \in A} \exp \left(-\beta H_{\Lambda}(\omega)\right) \\
& \quad=\frac{1}{Z} \sum_{\omega \in A} \exp \left(-\beta\left(H_{\Lambda}(\omega)-H_{\Lambda}(\omega \backslash\{e\})\right) \exp \left(-\beta H_{\Lambda}(\omega \backslash\{e\})\right)\right. \\
& \leq \exp \left(-\beta L(e)-\beta\left(h_{1}-h_{0}\right)\right) \frac{1}{Z} \sum_{\omega \in A} \exp \left(-\beta H_{\Lambda}(\omega \backslash\{e\})\right) \\
& \leq \exp \left(-\beta L(e)-\beta\left(h_{1}-h_{0}\right)\right) \mathrm{P}_{\Lambda}\left(e \notin \omega \mid \omega \cap B_{\gamma}=\xi\right) \\
& \leq \exp \left(-\beta L(e)-\beta\left(h_{1}-h_{0}\right)\right) .
\end{aligned}
$$

The event $\{e \in \omega\}$ is cylindrical and so is $\left\{\omega \cap B_{\gamma}=\xi\right\}$, as $B_{\gamma}$ is finite by hypothesis. Hence we can take the limit as $\Lambda \uparrow \mathbb{R}^{d}$ and conclude.

Proof of Theorem 5: Without losing generality we assume that the origin is a point of $\Gamma, 0 \in \Gamma$. Let $C(\omega)$ be the vertex set of the graph $C_{0}(\omega)$ and $E(\omega)$ its edge set. Let us show that under the conditions of the theorem the expected number of vertices in the open cluster of the origin $\mathrm{E}|C|$ is finite. This is inspired in the method of generations introduced by Menshikov ${ }^{6}$ to show no percolation in the site percolation model.

For a given configuration $\omega$ let $E(\omega)$ be the edge set of the open cluster $C(\omega)$, let $V_{0}(\omega)=\{0\}$ and for $n \geq 1$ define

$$
\begin{aligned}
& V_{n}(\omega):=\left\{\gamma^{\prime} \in \Gamma \backslash\left(V_{0}(\omega) \cup \cdots \cup V_{n-1}(\omega)\right):\right. \\
& \text { there is a } \left.\gamma \in V_{n-1}(\omega) \text { such that } \gamma \gamma^{\prime} \in E(\omega)\right\}, \\
& O_{n}(\omega):=\left\{\gamma \gamma^{\prime} \in E(\omega): \gamma \in V_{n-1}(\omega), \gamma^{\prime} \in V_{n-1}(\omega) \cup V_{n}(\omega)\right\} .
\end{aligned}
$$

Each vertex in $V_{n}(\omega)$ is attained with at least a path of $n$ distinct open edges starting from the origin but cannot be attained with a shorter open path. Each edge in $O_{n}(\omega)$ is the $n$-th step of an open self avoiding path starting from the origin and not belonging to a shorter open path. Since $\left(V_{n}, n \geq 0\right)$ is a partition of the set of vertices of the open cluster at 0 ,

$$
\mathrm{E}|C|=\sum_{n=0}^{\infty} \mathrm{E}\left|V_{n}\right| .
$$

If we show that for $\varepsilon$ defined in (8) and $n \geq 0$,

$$
\mathrm{E}\left|V_{n+1}\right| \leq \varepsilon \mathrm{E}\left|V_{n}\right|,
$$

then the sum in (16) is bounded by $\frac{\varepsilon}{1-\varepsilon}$ and the theorem is proven. 
Proof of (17): $\quad$ Call $\widetilde{O}_{n}(\omega):=O_{1}(\omega) \cup \ldots \cup O_{n}(\omega)$. The edges of the $(n+1)$-th generation is the union of the edges belonging to the stars centered at the points of $V_{n}$ and not contained in previous generations:

$$
O_{n+1}(\omega)=\bigcup_{\gamma \in V_{n}(\omega)}\left(\omega \cap S_{\gamma} \backslash \widetilde{O}_{n}(\omega)\right)
$$

For $n \geq 1$ define

$$
F_{n}(\omega):=\left\{\gamma \gamma^{\prime} \in E \backslash E(\omega): \gamma \in V_{n-1}(\omega), \gamma^{\prime} \in \Gamma\right\}
$$

the set of closed edges incident to the vertices in $V_{n-1}$. Let $\widetilde{F}_{n}:=F_{1} \cup \cdots \cup F_{n}$.

Let $\xi$ be a possible set of open edges for the first $n$ generations: $\xi=\widetilde{O}_{n}(\xi)$. For any $\omega$, if $\xi=\widetilde{O}_{n}(\xi)=\widetilde{O}_{n}(\omega)$, then $\widetilde{F}_{n}(\xi)=\widetilde{F}_{n}(\omega)$. Since every vertex has finite degree, the set of possible $\xi$ is countable and

$$
\sum_{\xi} \mathrm{P}\left(\widetilde{O}_{n}(\omega)=\xi\right)=1
$$

where the sum runs over finite $\xi \subset E$ such that $\xi=\widetilde{O}_{n}(\xi)$.

Fix $\gamma \in V_{n}(\xi)$ and let $B_{\gamma}(\xi):=S_{\gamma} \cap\left(\widetilde{O}_{n}(\xi) \cup \widetilde{F}_{n}(\xi)\right)$, the set of edges incident to $\gamma$ already determined by $\xi$ and $D_{\gamma}(\xi):=S_{\gamma} \backslash B_{\gamma}(\xi)$ the edges in $S_{\gamma}$ that are not fixed by $\xi$. Since $\xi$ is finite, so is $B_{\gamma}(\xi)$. Take $e \in D_{\gamma}(\xi)$. Then,

$$
\begin{aligned}
\mathrm{P}\left(e \in \omega \mid \widetilde{O}_{n}(\omega)=\xi\right) & =\mathrm{P}\left(e \in \omega \mid \omega \cap B_{\gamma}(\xi)=\xi \cap B_{\gamma}(\xi)\right) \\
& \leq \exp \left(-\beta L(e)-\beta\left(h_{1}-h_{0}\right)\right),
\end{aligned}
$$

by Lemma 4; indeed $\xi \cap B_{\gamma}(\xi)$ is not empty. The obtained bound does not depend on $\xi$. Since $\left|V_{n}(\xi)\right| \leq\left|O_{n}(\xi)\right|$ for all $\xi$ and $n \geq 1$,

$$
\begin{aligned}
\mathrm{E}\left(\left|V_{n+1}\right| \mid \widetilde{O}_{n}(\omega)=\xi\right) & \leq \mathrm{E}\left(\left|O_{n+1}\right| \mid \widetilde{O}_{n}(\omega)=\xi\right) \\
& =\sum_{\gamma \in V_{n}(\xi)} \sum_{e \in D_{\gamma}(\xi)} \mathrm{P}\left(e \in \omega \mid \widetilde{O}_{n}(\omega)=\xi\right) \\
& \leq \sum_{\gamma \in V_{n}(\xi)} \sum_{e \in E_{\gamma}} \exp \left(-\beta L(e)-\beta\left(h_{1}-h_{0}\right)\right) \\
& \leq T(\beta) \exp \left(-\beta\left(h_{1}-h_{0}\right)\right)\left|V_{n}(\xi)\right|=\varepsilon\left|V_{n}(\xi)\right| .
\end{aligned}
$$

Hence,

$$
\mathrm{E}\left|V_{n+1}\right|=\sum_{\xi} \mathrm{E}\left(\left|V_{n+1}\right| \mid \widetilde{O}_{n}(\omega)=\xi\right) \mathrm{P}\left(\widetilde{O}_{n}(\omega)=\xi\right) \leq \varepsilon \mathrm{E}\left|V_{n}\right|
$$

\section{FINAL REMARKS}

Since the ground state of Gibbs Random Graph does not percolate, the theorems about nonpercolation show a kind of "stability" of the ground states.

Sufficient conditions for the existence of an infinite open cluster and monotonicity of $\theta_{\gamma}$ as function of $\beta$ (or $(\lambda, \beta))$ are open problems.

\section{ACKNOWLEDGMENTS}

The work of E.A.P. was partly supported by CNPq grants 300576/92-7 and 662177/96-7, (PRONEX) and FAPESP grant 99/11962-9, RFBR grants 07-01-92216 and 08-01-00105.

The work of V.V.S. was supported by FAPERJ (grants E-26/170.008/2008 and E26/110.982/2008) and CNPq (grants 471891/2006-1, 309397/2008-1 and 471946/2008-7). 
The work of A.Ya. was partly supported by FAPERJ grant E26/170.008/2008, and CNPq 306092/2007-7.

${ }^{1}$ B. Bollobás, Random Graphs, 2nd ed. (Cambridge University Press, Cambridge, 2001), vol.73.

${ }^{2}$ R. Durrett, Random Graph Dynamics (Cambridge University Press, Cambridge, 2007).

${ }^{3}$ H.-O. Georgii, O. Häggström, and C. Maes, "The random geometry of equilibrium phases." In phase transitions and critical phenomena, Vol. 18. Phase Transit. Crit. Phenom., vol. 18. Academic Press, San Diego, CA, 1-142.

${ }^{4}$ G. R. Grimmett, and D. J. A. Welsh, Disorder in Physical Systems. edited by (The Clarendon Press Oxford University Press, New York, 1990).

${ }^{5}$ R. Meester, and R. Roy, Continuum percolation. Cambridge Tracts in Mathematics (Cambridge University Press, Cambridge, 1996), vol. 119.

${ }^{6}$ M. V. Men'shikov, S. A. Molchanov, and A. F. Sidorenko, (1986). Percolation theory and some applications. In Probability theory. Mathematical statistics. Theoretical cybernetics, Vol. 24 (Russian). Itogi Nauki i Tekhniki. Akad. Nauk SSSR Vsesoyuz. Inst. Nauchn. i Tekhn. Inform., Moscow, 53-110, i. Translated in J. Soviet Math. 42(4), 1766 (1988). 